\title{
Concurrent upper and lower urinary tract robotic surgery: A case series
}

\author{
Sailaja Pisipati, MD, FRCS (Urol); Christian Bach, MD, FRCS (Urol); Datesh Daneshwar, MD, FRCS (Urol); \\ Edward W. Rowe, MD, FRCS (Urol); Anthony J. Koupparis, MD, FRCS (Urol)
}

Department of Urology, Bristol Urological Institute, Southmead Hospital, Bristol, United Kingdom

Cite as: Can Urol Assoc J 2014;8(11-12):e853-8. http://dx.doi.org/10.5489/cuaj.1836 Published online November 24, 2014.

\section{Abstract}

The da Vinci Surgical System (Intuitive Surgical Inc.) continues to develop as a platform in urological surgery. Synchronous upper and lower urinary tract tumours requiring extirpative surgery are not uncommon. We report the first case robotic series of combined complex upper and lower urinary tract surgery. Six high-risk anaesthetic patients with a median age of 71 years and apparent synchronous upper and lower urinary tract pathologies underwent concurrent robotic surgery. Five underwent robotic nephroureterectomy and robotic-assisted radical cystectomy (RARC); 1 had combined robotic nephroureterectomy and robotic-assisted radical prostatectomy (RALP). The mean length of stay was 10 days, with an average blood loss of $416.7 \mathrm{~mL}$. The median console time for nephroureterectomy, RALP and RARC was 90, 90 and 210 minutes, respectively. Four patients had intra-corporeal ileal conduit urinary diversion. There were no Clavien grade 3, 4, or 5 complications. In all patients, 30- and 90-day mortality was nil. Margins were clear in the entire cohort. We concluded that combined upper and lower urinary tract robotic surgery is safe and technically feasible with acceptable complications and oncological outcomes.

\section{Introduction}

The da Vinci Surgical System (Intuitive Surgical Inc.) continues to develop as a platform in urological surgery. Its use has been described in both pelvic and upper tract uro-oncological procedures. Synchronous upper and lower urinary tract tumours requiring extirpative surgery are not uncommon. Traditionally these have been managed with extensive open surgery or a combination of minimally invasive upper tract and open pelvic surgery. These are performed either concurrently, but more frequently, as two separate procedures. We report the first robotic case series of combined complex upper and lower urinary tract surgery. This surgery involves synchronous removal of the kidney and the ureter, along with the prostate and/or bladder. We propose that this procedure is technically feasible and safe to manage concomitant upper and lower urinary tract cancer.

\section{Methods}

Of the 6 patients in this case series who underwent combined upper and lower urinary tract robotic surgery, 5 had cardio-pulmonary exercise testing (CPEX) preoperatively for assessment of risk and appropriate counselling. Patients were anesthetized and positioned in the appropriate lateral position following urinary catheterization. Pressure points were protected using full-length gel pads. The operating table was fully flexed and patients were secured with straps and wide adhesive tape.

\section{Port placement and technique for upper tract robotic surgery}

An open Hassan technique was used to place the 12-mm camera port lateral and cranial to the umbilicus and $a$ pneumo-peritoneum of $12 \mathrm{mmHg}$ was established for instrument port placement. Two $8-\mathrm{mm}$ robotic instrument ports were triangulated about $8 \mathrm{~cm}$ from the camera port. A 5-mm assistant port was placed caudal and lateral to the xiphoid for liver retraction. A second 12-mm assistant port was placed cranial and slightly medial to the camera port (Fig. 1). The robot was docked posteriorly at an approximate 20-degree angle towards the head of the patient.

The nephroureterectomy portion of the procedure was performed in the usual way, as with the laparoscopic approach, with hepatic retraction, colonic and lower renal polar mobilization and early control of the ureter. The ureter was then followed cranially to identify and control the renal hilar vasculature. The remaining renal attachments were divided to facilitate the mobilization of the entire kidney. Following this, the ureter was dissected caudally to the level of crossing of the common iliac artery. A Hemolock clip 
with a vicryl tie attached was applied to the ureter at this level; this enabled us to identify and retract the ureter after repositioning the patient for lower tract surgery.

\section{Port placement and technique for robotic-assisted laparoscopic pros- tatectomy}

The patient was then repositioned in the lithotomy position with an extreme Trendelenburg tilt. For patients with a smaller body habitus, we used the same camera port site and the most caudal of the $8-\mathrm{mm}$ robotic port sites for the lower tract surgery (Fig. 1, Fig. 2). Otherwise, all of the port sites were closed formally and ports were re-positioned as previously described for robotic prostatectomy. ${ }^{1}$

The technique for robotic-assisted laparoscopic prostatectomy (RALP) and pelvic lymphadenectomy has been well-described..$^{1-3}$ We adopted this technique with a slight modification while performing concurrent upper and lower tract surgery. The left ureter was dissected further distally and a cuff of bladder was partially dissected. The final release of the ureteric orifice and reconstruction were performed concurrently with the anterior and posterior bladder neck dissection. We found that bladder reconstruction is best performed prior to the release of lateral prostatic pedicles as exposure to the bladder defect is better at this point rather than after the prostate has been removed.

\section{Port placement and technique for robotic-assisted radical cystectomy}

Following re-positioning of the patient, the same camera port and the most distal of the 8-mm robotic port sites were used

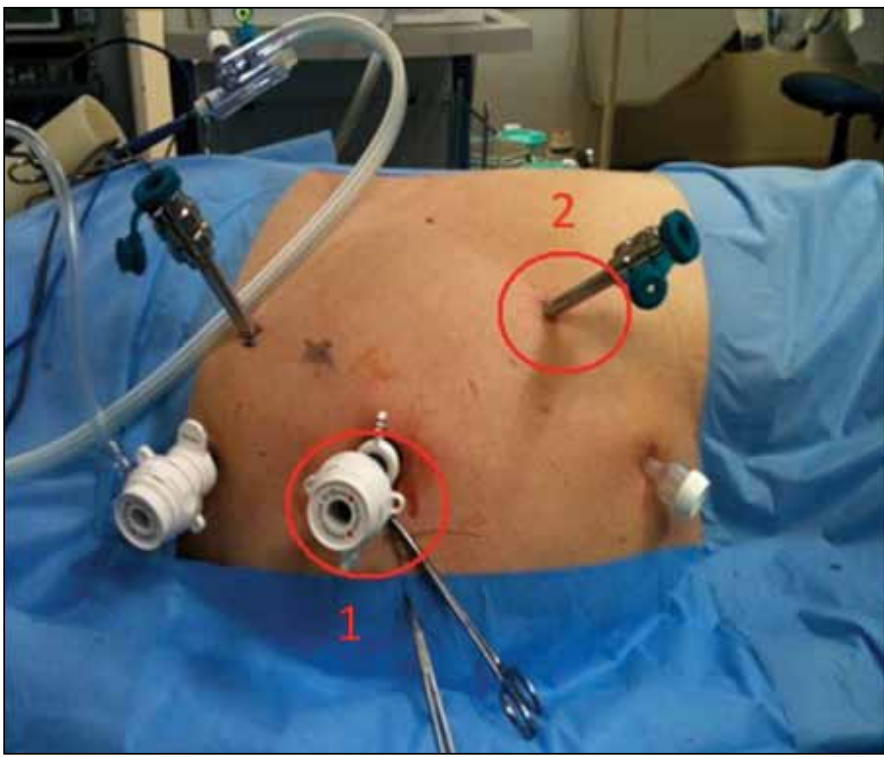

Fig. 1. Port placement for upper urinary tract robotic surgery. 1: 12-mm camera port; 2: 8-mm robotic instrument port. for lower tract surgery (Fig. 1, Fig. 2) in patients with a small body habitus. If this was not feasible due to the body habitus, ports for robotic cystectomy were positioned as previously described ${ }^{4}$ following closure of all the existing port sites. Our robotic approach to the bladder for robotic-assisted radical cystectomy (RARC) started with identification of the ureters (Fig. 3) and an extended pelvic lymphadenectomy bilaterally (Fig. 4). Following proximal and distal ureteric mobilization, the ureters were clipped and divided bilaterally. This then facilitated excellent visualization during dissection of the lateral vesicle pedicles and bladder mobilization. The third phase of RARC was the intra-corporeal urinary diversion, which in the current cohort of patients were ileal conduits (Fig. 5, Fig. 6).

Cystectomy specimen retrieval was achieved through an extension of the camera port in men and through the vagina in women, thereby avoiding the need for further incisions. A urethral catheter into the free pelvis acted as a drain.

The combined robotic approach involved repositioning the patient and undocking and re-docking the da Vinci robot. Although repositioning the patient did not take long, re-arranging the robotic set-up between the upper tract and lower tract parts of the procedure required planning. The presence of experienced scrub staff and a trained physician's assistant familiar with the technique was as critical as having a skilled surgeon and anesthetist in the operating room, for an uneventful procedure.

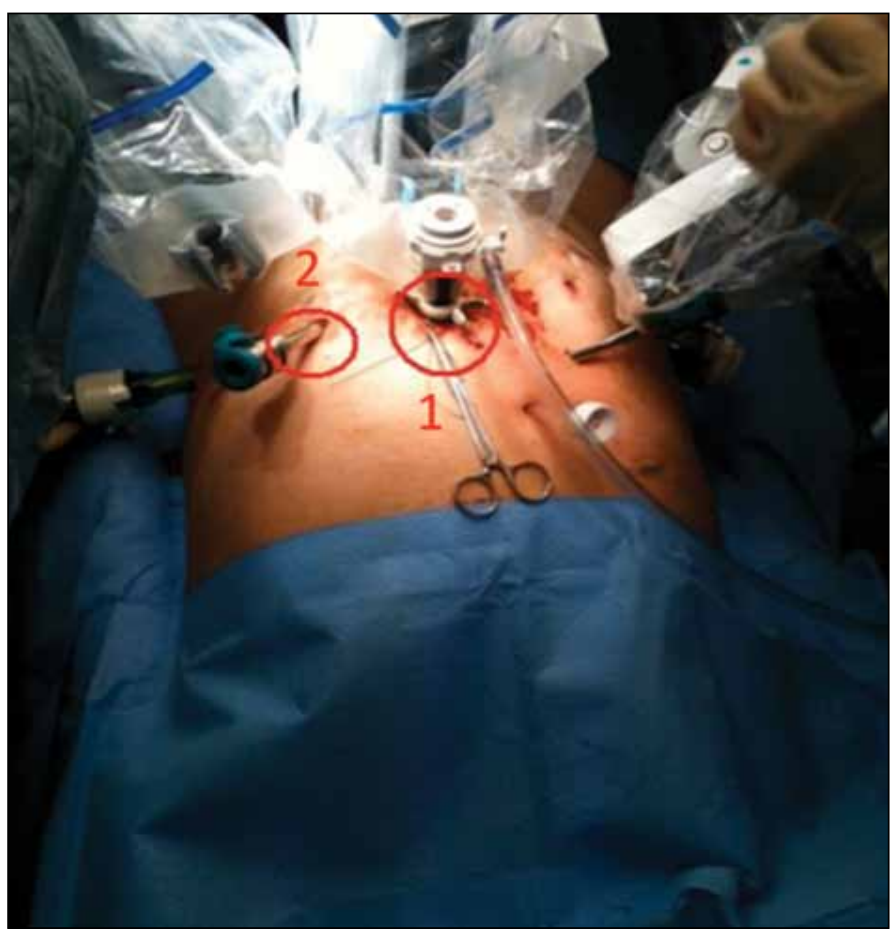

Fig. 2. Port placement for lower urinary tract robotic surgery. In suitable candidates, the same 12-mm camera port (1) and an 8-mm robotic port (2) can be used for both upper and lower tract robotic surgery. 

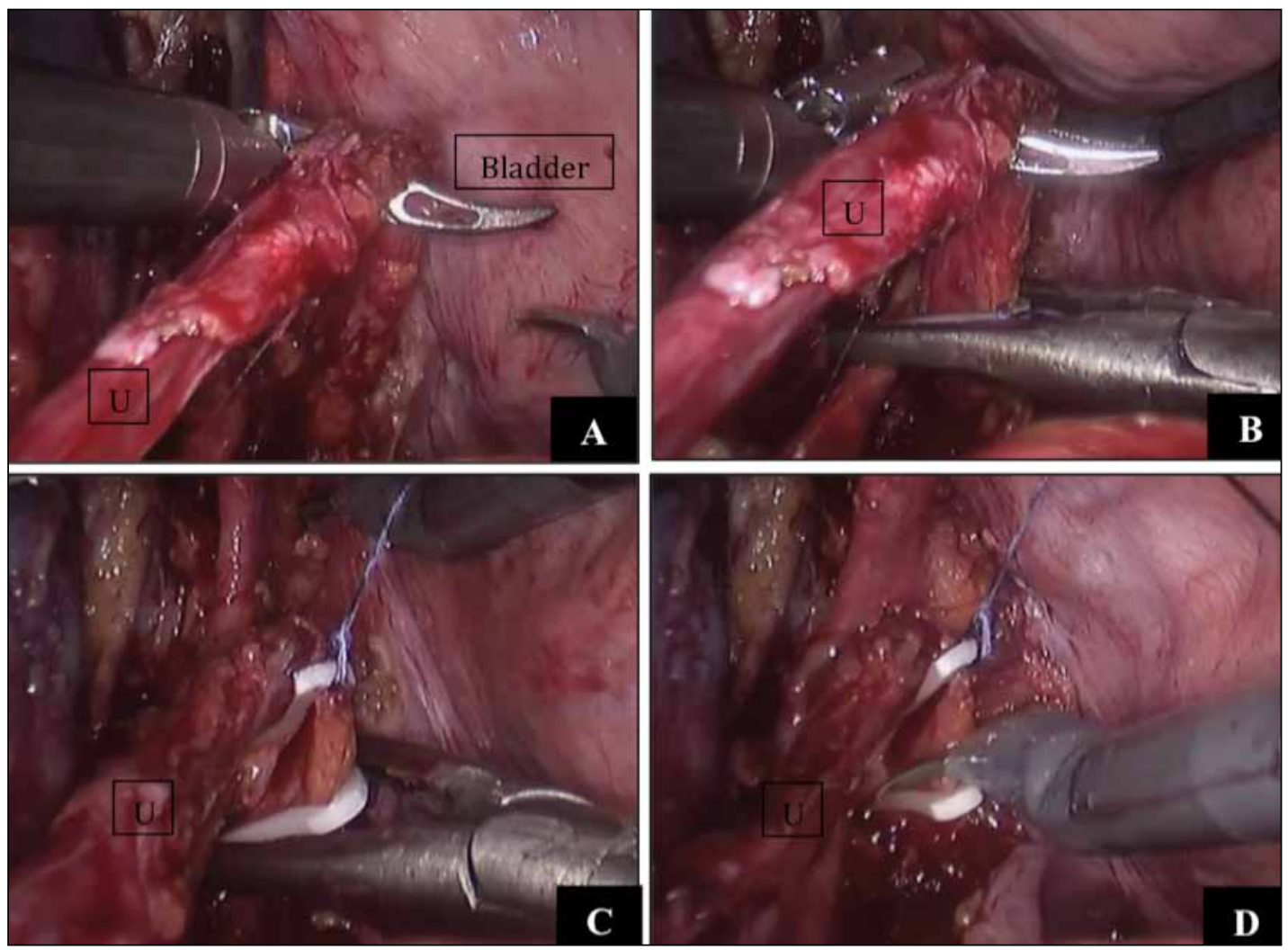

Fig. 3. A: Ureter (U) that has already been mobilized during upper tract surgery is identified. B, C, D: The ureter is clipped and divided bilaterally.

\section{Results}

In our series, 6 high-risk anesthetic patients (1:1 male-female ratio) with apparent synchronous upper and lower urinary tract abnormalities underwent concurrent robotic surgery. The first patient in our series had a robotic nephroureterectomy and RALP, and the subsequent 5 had robotic nephroureterctomy and RARC with ileal conduit urinary diversion. The robotic nephroureterectomy on the first patient in this series was challenging due to prior open ureteric stone extraction. None of the other patients had prior abdominal surgery. Four of the 5 patients who had combined nephroureterectomy and RARC had an intra-corporeal approach for urinary diversion. Nerve-sparing RALP was performed. None of the patients who had RARC had a formal nerve-sparing procedure in view of their poor preoperative sexual function (Table 1).

All the patients who had RARC were on our enhanced recovery after major surgery program. This involved a proactive role from a multidisciplinary team consisting of medical staff, cancer nurse specialists, nursing staff on the wards, pain team, physiotherapists, dieticians, and stoma care team. The members of this team not only ensured adequate preoperative preparation and counselling, perioperative dietary advice, adequate pain relief, early mobilization, venous thromboembolic prophylaxis and bowel care, but also emotional, psychological and social support to motivate the patient. The overall goal was to provide a high standard of care by adopting a multidisciplinary approach, thereby minimizing complications and facilitating early mobilization and discharge. As per this program, our patients were allowed low residue solid diet up to 6 hours before surgery and were given high-calorie drinks up to 3 hours preoperatively. No bowel preparation was provided. Following surgery, patients were encouraged to sit out on postoperative day (POD) 1 and ambulate on POD 2. Clear fluids were allowed on the day of surgery, free fluids on POD 1 and soft diet on POD 1 or 2 as tolerated. Patients were encouraged to use chewing gum. We observed that avoiding bowel preparation, early ambulation, chewing gum and early resumption of oral intake resulted in early return of bowel function between POD 2 and 4.

\section{Discussion}

Despite the successful performance of minimally invasive nephroureterectomy/nephrectomy and radical cystectomy as separate surgeries, there is limited data on concurrent execution of these procedures. ${ }^{5}$ Minimally invasive laparoscopic procedures can be used in most patients with urological neoplasms, including patients with synchronous primary uro- 

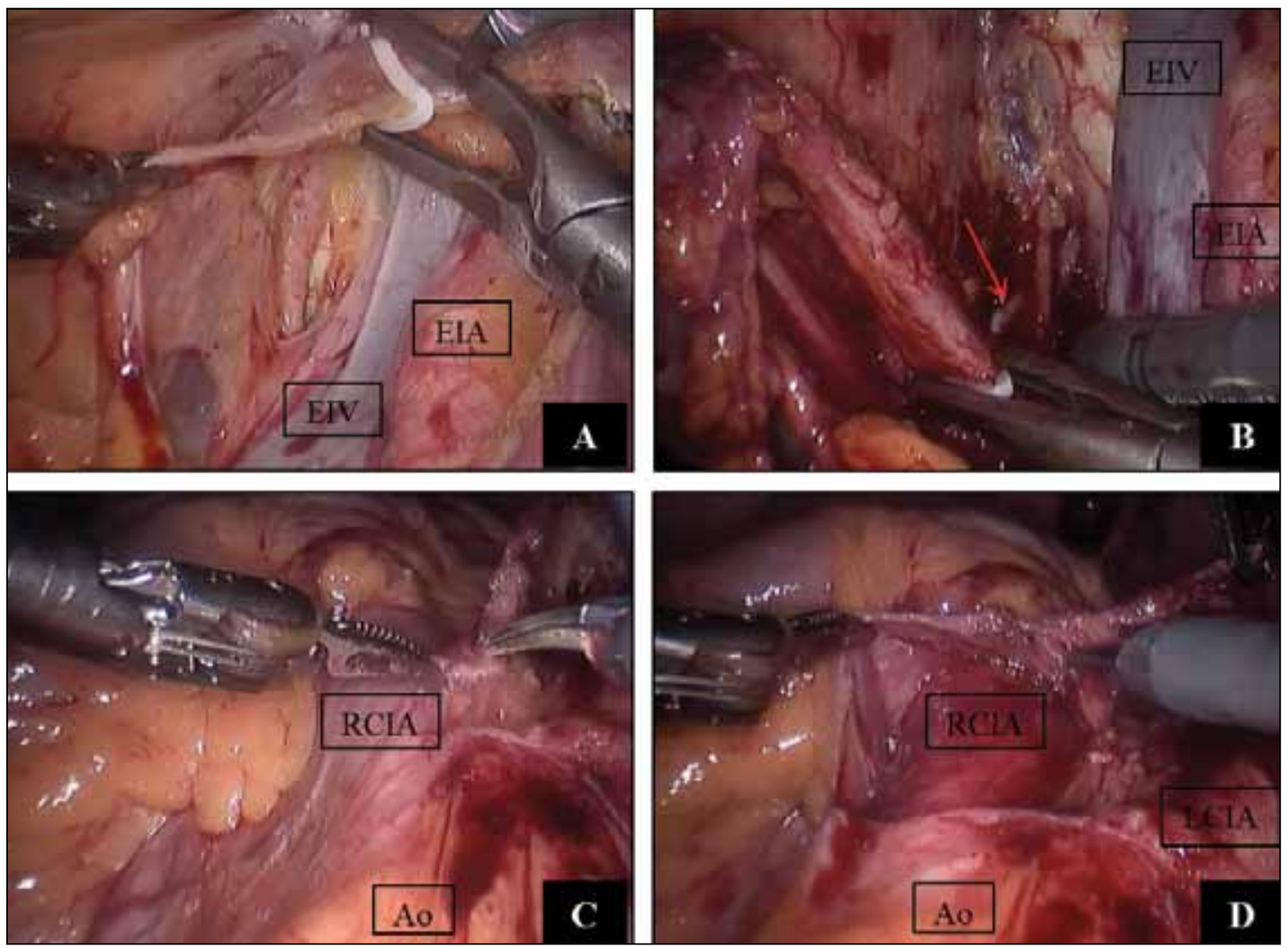

Fig. 4. Extended pelvic lymphadenectomy. A, B: External iliac and obturator lymphadenectomy. C, D: Common iliac lymphadenectomy. EIA: external iliac artery; EIV: external iliac vein; RCIA: right common iliac artery; LCIA: left common iliac artery; Ao: aorta. Red arrow points to obturator nerve.

logic malignancies. ${ }^{6-8}$ Additional reports have been described for combined surgery in patients with bladder cancer and concurrent end-stage renal disease. ${ }^{9-11}$ Reports of combined upper and lower urinary tract robotic procedures are scarce.

Robotic-assisted laparoscopic surgery offers patients a minimally invasive treatment option. Concurrent upper and lower urinary tract robotic surgery offers a few advantages. These include avoidance of multiple surgeries and anesthetics for the patient thus reducing the morbidity. It also eliminates the time, cost and convalescence associated with a second operative procedure and hospital admission. Although, this translates to longer operative times, our case series demonstrates acceptable complication rates.

In their feasibility study of combined upper and lower urinary tract robot-assisted laparoscopic surgery, Eun and colleagues described a 4-port baseball diamond strategy to allow instrument access without repositioning the patient and re-docking the robot. Their novel pivoting triangle
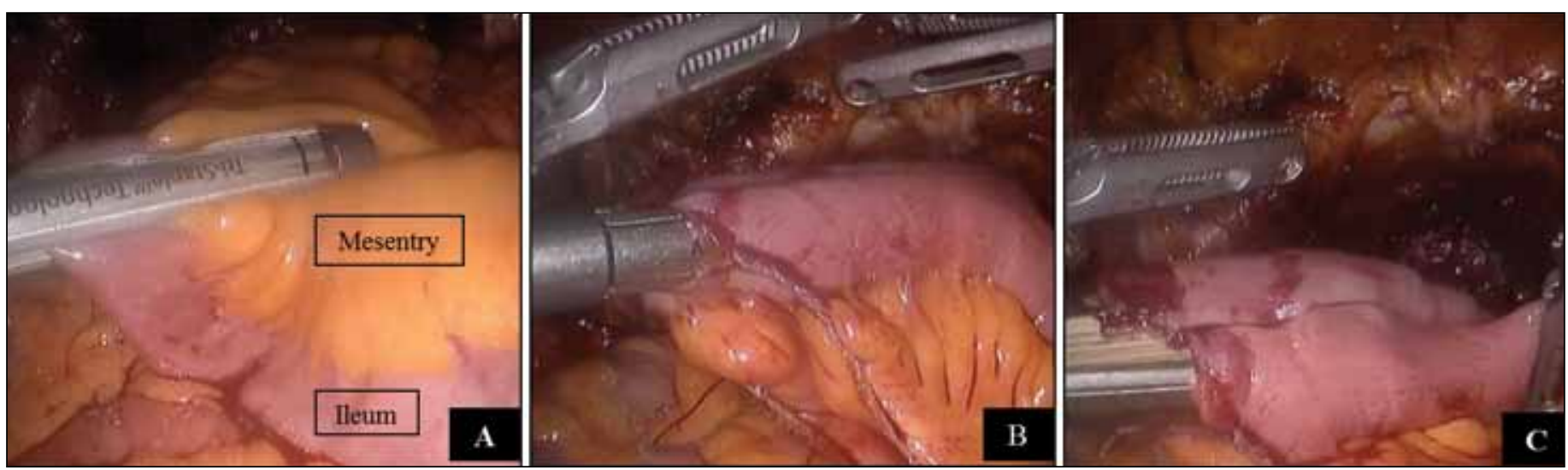

Fig. 5. A loop of ileum is separated from the rest of the small bowel $(A)$ and $(B, C)$ side to side stapled small bowel anastomosis is performed with to restore continuity. 
manoeuvre allowed dual port cannulation and lens telescoping by incorporating the fourth arm, thus providing additional length to reach the deep pelvis. ${ }^{12}$ Cases of RALP combined with robotic-assisted trans-peritoneoscopic and retro-peritoneoscopic partial nephrectomy have been previously reported. ${ }^{1,2}$ Finley and colleagues described the initial report of combined RALP and hand-assisted laparoscopic nephroureterectomy with robotic assistance and reported the novel use of the da Vinci robot for extravesical bladdercuff excision and repair. ${ }^{3}$ Following this, Lavery and colleagues published the first report on successful combined RALP and robotic radical nephrectomy to treat synchronous prostatic adenocarcinoma and clear-cell renal cell carcinoma. ${ }^{13}$ Also, Benabdallah and colleagues have reported their initial report of robot-assisted laparoscopic combined nephroureterectomy and cystoprostatectomy where pelvic lymph node dissection and urinary diversion with an ileal conduit were performed extra-corporeally. ${ }^{5}$

Promising reports on the outcomes of robotic nephroureterectomy are slowly emerging. ${ }^{14-17}$ Although pure robotic nephroureterectomy incorporates both upper and lower tract dissection, it would not be ideal to directly compare
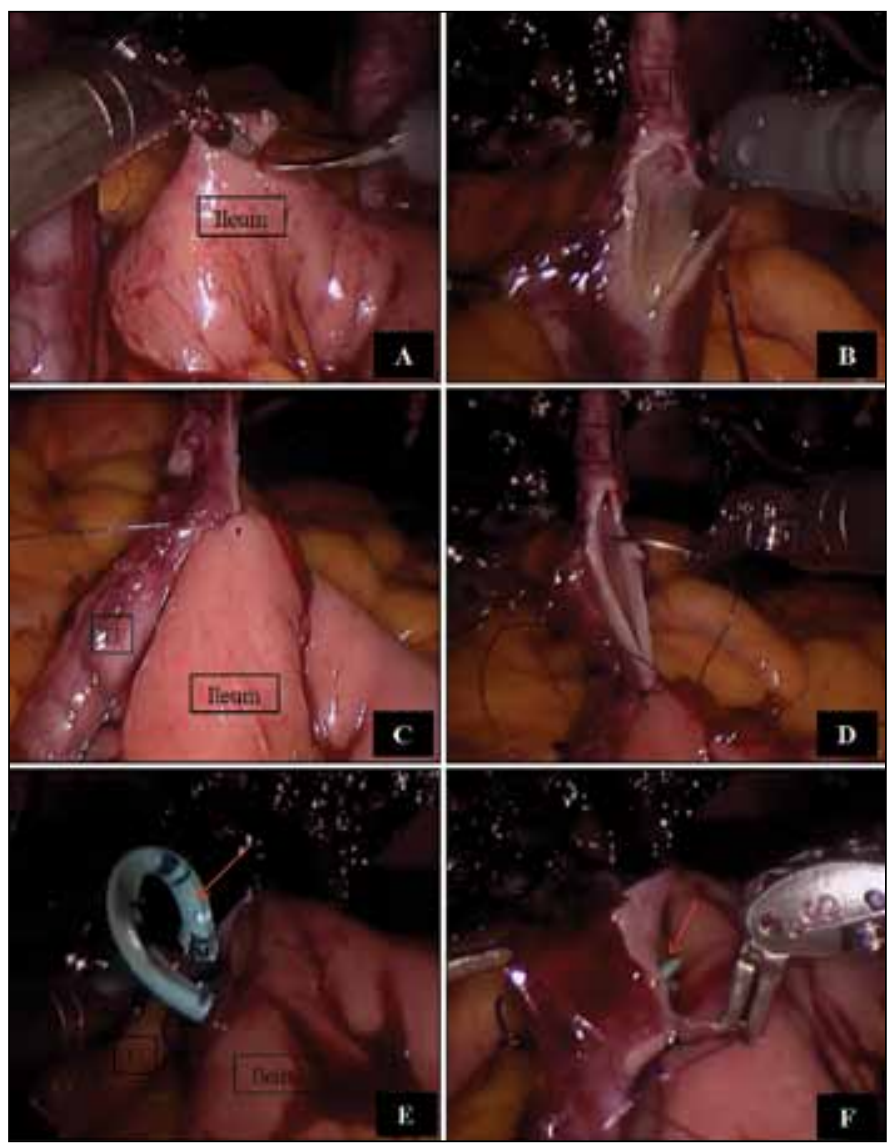

Fig. 6. Uretero-ileal anastomosis. A: Ileal enterotomy is performed. B:

Ureterotomy performed. C, D: Bricker uretero-ileal anastomosis is performed.

$\mathrm{E}, \mathrm{F}$ : Uretero-ileal anastomosis is completed over a ureteric stent (red arrows).
Table 1. Results from combined upper and lower urinary tract robotic surgery case series

\begin{tabular}{lc}
\hline Parameter recorded & Results \\
\hline No. patients & 6 \\
Mean age, year (range) & $71(61-77)$ \\
Mean ASA & 2.3 \\
Average LOS, days (range) & \\
Robotic nephroureterectomy and RALP & 4 \\
Robotic nephroureterectomy and RARC & $10.2(5-14)$ \\
Average blood loss, mL & 416.7 \\
Combined robotic procedure, n & \\
Robotic nephroureterectomy and RALP & 1 \\
Robotic nephroureterectomy and RARC & 5 \\
Median console time, min (range) & \\
Nephroureterectomy & $90(55-240)$ \\
RALP & 90 \\
RARC & $210(200-240)$
\end{tabular}

Urinary diversion, $\mathrm{n}$

lleal conduit

Technique of urinary diversion, $\mathrm{n}$

Intra-corporeal

Extra-corporeal

Upper tract histology, $\mathrm{n}$

Non-functioning kidney

Multiple oncocytomas

T2c G3 ureteric TCC with squamous differentiation

T3 G3 ureteric TCC

TO Benign (preoperative CT scan revealed filling defects)

Postoperative bladder histology and pathological

stage, $n$

pT0

pT1 G3 TCC

pT3b G3 TCC + CIS

pT3b SCC

Lymph node involvement, $\mathrm{n}$

N1

Histology from RALP specimen $(n=1)$

pT2c

Gleason grade

Incidental prostate cancer, $\mathrm{n}$

Histology from incidental prostate cancer specimen

pT2a

Gleason grade

Clavien complications, $\mathrm{n}$

Grade 2

Grade 3

Grade 4

Grade 5

Margins, $n$

Clear

30-day mortality

90-day mortality

ASA: American Society of Anesthesiologists; LOS: length of stay; RALP: robotic-assisted radical prostatectomy; RARC: robotic-assisted radical cystectomy; TCC: transitional cell carcinoma; SCC: CIS: carcinoma in situ; CT: computed tomography. 
Pisipati et al.

the results of those series with our current series due to the inclusion of RARC, which is probably the most complex and challenging procedure in urological surgery.

We report the first ever case series of combined roboticassisted nephroureterectomy/nephrectomy and RARC. Four of the 5 patients who underwent combined nephroureterectomy and RARC had intra-corporeal urinary diversion. The length of hospital stay reduced from 13 days following open radical cystectomy ${ }^{18}$ to 10 days following combined robotic nephroureterectomy and RARC. There were no complications of Clavien grade 3 or greater. The use of the da Vinci robot for en bloc excision of the kidney, ureter, bladder and the prostate is technically feasible, safe and conforms to oncological principles. ${ }^{5}$ With increasing experience, surgical times could improve. The authors accept that this series involves a small cohort of patients with a short follow-up. This article focusses on the perioperative outcomes and our primary aim was to convey that combined upper and lower tract robotic surgery, although complex and challenging, is technically feasible.

\section{Conclusion}

Single session, concurrent multiple quadrant robotic urological surgery in high-risk anesthetic patients is facilitated and feasible by using the da Vinci Surgical System with acceptable complications and oncological outcomes. In carefully selected patients with thorough preoperative assessment, the combined robotic approach can reduce the morbidity, complications, hospital admissions and total period of convalescence.

Competing interests: All authors declare no competing financial or personal interests.

This paper has been peer-reviewed.

\section{References}

1. Patel MN, Eun D, Menon M, et al. Combined robotic-assisted laparoscopic partial nephrectomy and radical prostatectomy. ISLS 2009;13:229-32.

2. Guttilla A, Crestani A, Zattoni F, et al. Combined robotic-assisted retroperitoneoscopic partial nephrectomy and extraperitoneal prostatectomy. First case reported. Urologia 2012;79:62-4. http://dx.doi. org $/ 10.5301 /$ RU.2011.8884

3. Finley DS, Melamud 0, Omstein DK. Combined robot assisted laparoscopic nephroureterctomy and radical prostatectomy. J Endourol 2007;21:411-4. http://dx.doi.org/10.1089/end.2007.0013

4. Annerstedt $M$, Wiklund $P$. Step by step approach to robotic cystectomy and intra-corporeal urinary diversion. In: Patel VR, ed. Robotic Urological Surgery. 2nd ed. London, England: Springer; 2012:369-80.

5. Benabdallah $\mathrm{JO}$, Hampton $\mathrm{U}$, Guruli $\mathrm{G}$, et al. Robot-assisted laparoscopic combined nephroureterctomy and cystoprostatectomy: An initial report and review of the literature. J Robotic Surg 2012;6:159-62. http://dx.doi.org/10.1007/s11701-011-0273-8

6. Deng DY, Meng MV, Grossfield GD, et al. Simultaneous laparoscopic management of three urological malignancies. J Urol 2002;167:2125-6. http://dx.doi.org/10.1016/S0022-5347(05)65101-4

7. Oda J, Landman J, Bhayani S, et al. Concomitant laparoscopic hand-assisted radical nephrectomy and open radical prostatectomy using a single lower midline incision. Urology 2000;56:1056. http://dx.doi. org/10.1016/S0090-4295(00)00809-8

8. Barros R, Frota R, Stein RJ, et al. Simultaneous laparoscopic nephroureterectomy and cystectomy: A preliminary report. Int Braz J Urol 2008;34:413-21. http://dx.doi.org/10.1590/S167755382008000400003

9. Holton MR, Van Zili PS, Oberle WT, et al. Complete urinary tract extirpation: The University of Maryland experience. Urology 2006;68:65-9. http://dx.doi.org/10.1016/j.urology.2006.01.045

10. Wu CF, Shee JJ, Ho DR, et al. Different treatment strategies for end stage renal disease in patients with renal cell carcinoma. J Urol 2004;171:126-9. http://dx.doi.org/10.1097/01.ju.0000101758.41635.28

11. LiCC, Wang HS, Wu WJ, et al. Laparoscopic complete urinary tract exenteration with the specimen withdrawn transvaginally. BJU Int 2009;104:82-6. http://dx.doi.org/10.1111/i.1464-410X.2008.08339.x

12. Eun $D$, Bhandari $A$, Boris $R$, et al. Concurrent upper and lower urinary tract robotic surgery: Strategies for success. BJU Int 2007;100:1121-5.

13. Lavery $\mathrm{HJ}$, Patel $\mathrm{S}$, Palese $\mathrm{M}$, et al. Combined robotic radical prostatectomy and robotic radical nephrectomy. JSLS 2010;14:603-7. http://dx.doi.org/10.4293/108680810X12924466008763

14. Lim SK, Shin TY, Rha KH. Current status of robot assisted laparoscopic radical nephroureterectomy for management of upper tract urothelial carcinoma. Curr Urol Rep 2013;14:138-46. http://dx.doi. org/10.1007/s1 1934-012-0303-8

15. Ambani SN, Weizer AZ, Wolf IS Jr, et al. Matched comparison of robotic vs laparoscopic nephroureterectomy: An initial experience. Urology 2014;83:345-9. http://dx.doi.org/10.1016/i.urology.2013.07.079

16. Khemees TA, Nasser SM, Abaza R, et al. Clinical pathway after robotic nephroureterectomy: Omission of pelvic drain with next-day catheter removal and discharge. Urology 2014;83:818-23. http://dx.doi. org/10.1016/i.urology.2013.10.078

17. Zargar $\mathrm{H}$, Krishnan J, Autorino $\mathrm{R}$, et al. Robotic nephroureterectomy: A simplified approach requiring no patient repositioning or robot redocking. Eur Urol 2014;66:769-77. http://dx.doi.org/10.1016/i. eururo.2014.02.060

18. Arumainayagam N, McGrath J, Jefferson KP, et al. Introduction of an enhanced recovery protocol for radical cystectomy. BJU Int 2008;101:698-701. http://dx.doi.org/10.1111/i.1464-410X.2007.07319.x

Correspondence: Sailaja Pisipati, Urology Speciality Trainee, Southmead Hospital, Westbury-0n-Trym, Bristol, BS10 5NB, United Kingdom; sailaja13in@yahoo.com 